\title{
Niels Keiding 70 years
}

\author{
Per Kragh Andersen" ${ }^{1}$ Thomas H. Scheike ${ }^{1}$
}

Received: 24 June 2015 / Accepted: 17 August 2015 / Published online: 25 August 2015

(C) Springer Science+Business Media New York 2015

In May 2014, the Section of Biostatistics, University of Copenhagen, Denmark, in cooperation with the Danish Society of Theoretical Statistics, organised a 2-day symposium at the occasion of Niels Keiding's 70th birthday. A large audience was attracted by a programme including ten international speakers $(\varnothing$. Borgan, R. Henderson, R. Slama, E. Arjas, D. Commenges, V. Didelez, O.O. Aalen, N. Breslow, R.D. Gill, and D. Clayton) and four locals (T. Scheike, P.K. Andersen, E. Budtz-Jørgensen, and T. Martinussen). The lectures covered areas of Niels's research contributions, including survival analysis, causal inference, time-to-pregnancy, and epidemiological studies. Speakers highlighted the large impact that Niels has had on these research topics as well as his numerous contributions to the profession of biostatistics, both locally in Denmark and internationally.

International speakers were invited to contribute to this special issue of Lifetime Data Analysis and we are grateful for the smooth cooperation with Editor Mei-Ling Ting Lee and editorial assistant Divyalochany Thangavel during the process of editing this special issue.

Borgan (with R. Keogh) discusses how and when, in a nested case-control study, one can 'break the matching' and analyze the data using a pseudo-likelihood based on inverse probability of sampling weights. This may be particularly useful when the study includes several case series, however, more care is needed in the analysis due to the need for additional modeling of the effects of the matching variables. Therefore, the efficiency which may be gained should be balanced with the potential bias resulting.

\footnotetext{
$凶$ Per Kragh Andersen pka@biostat.ku.dk

Thomas H. Scheike ts@biostat.ku.dk

1 Section of Biostatistics, University of Copenhagen, Ø. Farimagade 5, PB 2099, 1014 Copenhagen, Denmark
} 
Henderson (with E. Elgmati, R.L. Fiaccone, and J.N.S. Matthews) presents methods for the analysis of discrete-time recurrent event data, when interest is mainly in prediction. When using flexible models such as the Aalen additive model, one problem is that one may encounter negative estimates. Alternatives, are the logistic regression model and penalized versions of it. The unpenalized version is unstable and with penalities there are bias. The authors therefore discuss the pros and cons of these methods, and suggest a modified penalized likelihood approach that makes a compromise between the penalized and unpenalized version of the logistic regression model. The methods are illustrated with two data sets.

D. Gasbarra (with Arjas, A. Vehtari, Slama, and Keiding) studies Bayesian inference for the so-called 'current duration design' which is a useful method in time-topregnancy studies. In this design, women currently attempting to become pregnant are followed from the time of sampling, utilizing also the time elapsed from initiation to sampling. A difficulty is that short waiting times to pregnancy are rarely selected into the sample but it is shown that a Bayesian estimation method provides a consistent estimator for the distribution. A simulation study illustrates the approach.

Commenges (with A. Gégout-Petit) presents a 'stochastic system' approach for estimation of (causal) effects of a dynamic treatment. Instead of relying on counterfactuals, the approach defines causality via the Doob-Meyer decomposition of a stochastic process. When this process develops in continuous time it becomes important to distinguish the underlying (continuous) process from the discrete-time observation process.

Aalen (with R.J. Cook and K. Røysland) elaborates on the 'hazard of hazard ratios' quote which is gaining some popularity in epidemiology. The paper discusses the potential causal interpretation of the standard hazard ratio estimated in a Cox regression model when based on data from a randomized trial. A main problem emphasized is that of unobserved prognostic variables which are balanced at baseline as a consequence of the randomization, but no longer so when some time in the trial has passed. It is highlighted that additive hazards models are not affected by this selection mechanism.

Breslow (with J. Hu, and J.A. Wellner) shows how Z-estimation can be applied for semiparametric models, in particular in the context of stratified sampling designs for censored survival data. Due to non-predictability of the weights in such an estimation procedure the authors rely on modern empirical processes to develop the properties of their estimators. Specifically, the authors consider estimation for the Cox proportional and Lin-Ying additive hazards models. Efficiency is improved by calibrating or estimating the weights using information available for all subjects. Although inefficient in comparison with likelihood inference for incomplete data, which is often difficult to implement, the approach provides consistent estimates of desired population parameters even under model misspecification.

All in all, the 6 articles provide a nice summary of what was presented at the symposium and, thereby, of areas in which Niels Keiding during his life-long career as a biostatistician has made important contributions. 